\title{
LINEAR INEQUALITIES AND SOME RELATED PROPERTIES OF FUNCTIONS*
}

\author{
BY L. L. DINES
}

Some years ago Lovitt $\dagger$ treated a problem concerning preferential voting. When expressed in analytic terms, the problem could be reduced to the consideration of a set of simultaneous inequalities of the form

$$
\left\{\begin{array}{c}
a_{11} x_{1}+a_{12} x_{2}+\cdots+a_{1 \nu} x_{\nu}>0, \\
a_{21} x_{1}+a_{22} x_{2}+\cdots+a_{2 \nu} x_{\nu}>0, \\
\cdot \cdot \cdot \cdot \cdot \cdot \cdot \cdot \cdot \cdot \\
a_{\mu 1} x_{1}+a_{\mu 2} x_{2}+\cdots+a_{\mu \nu} x_{\nu}>0,
\end{array}\right.
$$

where the coefficients $a_{i j}$ were given real constants, and permissible values for the $x_{j}$ were to be determined. In the particular problem mentioned $\nu$ was equal to 3 ; hence each of the inequalities could be given a simple geometric interpretation, and the resultant restriction upon the variables $x_{j}$ of the entire set (1) could be discussed clearly and vividly in geometric terms. But for $\nu>3$, a discussion of the system (1) obviously requires analytic methods. If one attempts such a discussion, he will almost certainly contrast the lack of available formal machinery, with the elegant theory of the system of equations which results from (1) if the inequality signs be replaced by equality signs. Yet it is obvious that for inequalities as for equations, the entire body of facts is inherent in the matrix of coefficients $A \equiv\left\|a_{i j}\right\|$.

It was these considerations which directed my thoughts along the lines which my remarks will follow this afternoon. I shall endeavor first to indicate how, by the introduction of certain suitable definitions, the treatment of a system of linear inequalities (1) can be made somewhat analogous to the treatment of

* An address read by invitation of this Society and the Mathematical Association of America, at their joint session, December 31, 1929.

$\dagger$ W. V. Lovitt, Preferential voting, American Mathematical Monthly, vol. 23, p. 363. 
the corresponding system of linear equations. The central feature of the latter treatment is, as you will recall, the rank of the matrix of coefficients. An analogous notion will be introduced, specially designed to apply to the inequalities, and will be called the inequality-rank or I-rank of the matrix. Postponing, for the moment, a precise definition of the notion, I will merely say that the $I$-rank, like the ordinary rank, is a well defined integral-valued function of the coefficients $a_{i j}$, and can have any one of the values $0,1,2, \cdots, \nu$. In terms of this notion can be stated the following theorem,* which is comparable to the fundamental theorem in the theory of linear equations.

THEOREM 1. A necessary and sufficient condition for the existence of a solution of the system of inequalities (1) is that the I-rank of the matrix $\left\|a_{i j}\right\|$ be greater than zero. If the I-rank of this matrix is $k(>0)$, then the system admits a solution in which $k-1$ of the $x_{j}$ are arbitrary.

The proof is of course a consequence of the definition, and you will be spared the details. It is desirable, however, to get before us the general idea of the $I$-rank.

To take the simplest situation first, suppose all the coefficients in some one column of the matrix are different from zero and have the same sign. Then the solution of the system may be obtained immediately. If, for example, the column in question is the first, the system may be reduced to one of the two forms

or

$$
x_{1}>-\sum_{j=2}^{\nu} \frac{a_{i j}}{a_{i 1}} x_{j}, \quad(i=1,2, \cdots, \mu),
$$

$$
x_{1}<-\sum_{j=2}^{\nu} \frac{a_{i j}}{a_{i 1}} x_{j}, \quad(i=1,2, \cdots, \mu),
$$

according as the coefficients in the first column are all positive or all negative. In either case the variables $x_{2}, x_{3}, \cdots, x_{\nu}$ can be assigned values at pleasure, and $x_{1}$ is only required to admit a finite number of values as lower bounds or upper bounds. Under these circumstances, that is, when all the elements in one column of the matrix $A$ have the same sign, the matrix will

* Annals of Mathematics, vol. 20, p. 193. 
be said to be I-definite with respect to that column. To say merely that a matrix is $I$-definite will imply that there is some column with respect to which it is $I$-definite.

Suppose now that with respect to some certain column, say the $r$ th column, the matrix $A$ is not $I$-definite. Then the elements in that column can be divided into three classes, namely,

those which are positive: $a_{p r}, p=p_{1}, p, \cdots, p_{P}$, those which are negative: $a_{n r}, n=n_{1}, n_{i}, \cdots, n_{N}$, those which are zero: $\quad a_{z r}, z=z_{1}, z_{2}, \cdots, z_{Z}$,

the number of elements in the respective classes being represented by $P, N$, and $Z$.

We form now a certain matrix of $\nu-1$ columns and $P N+Z$ rows, which shall be known as the I-complement of the rth column of the given matrix $A$. A typical column of this new matrix, the column characterized by the index $j$, consists of the elements

$$
\begin{gathered}
a_{p r} a_{n j}-a_{n r} a_{p j},\left\{\begin{array}{l}
\left(p=p_{1}, p_{2}, \cdots, p_{P}\right), \\
\left(n=n_{1}, n_{2}, \cdots, n_{N}\right),
\end{array}\right. \\
a_{z j}, \quad\left(z=z_{1}, z_{i}, \cdots, z_{Z}\right) .
\end{gathered}
$$

The $\nu-1$ columns are obtained by taking $j=1,2, \cdots, r-1$, $r+1, \cdots, \nu$.

If the given matrix $A$ is not $I$-definite with respect to any of its columns, there will be $\nu$ of these $I$-complement matrices. By suitable conventions to take care of the exceptional cases, we may properly say that there are always $\nu$ of them. They will be called the first $I$-minors or the $I$-minors of $\nu-1$ columns of the matrix $A$.

In order not to burden you with more detail, suffice it to say that each of the first $I$-minors so formed can be treated exactly as was the original matrix, and thus a system of second $I$-minors of $\nu-2$ columns defined. And the process may be repeated to determine successively $I$-minors having columns in number: $\nu-3, \nu-4, \cdots, 1$. We can now state the following definition.

Definition. A matrix will be said to be of I-rank $k$ if it possesses at least one I-minor of $k$ columns which is I-definite, but possesses no I-minor of $k+1$ columns which is I-definite. If none of its I-minors is I-definite it will be said to be of I-rank zero. 
It is in terms of this definition that Theorem 1 is to be interpreted.

Two additional remarks will suffice to make complete the study of linear algebraic inequalities from the point of view which I have been presenting. The first has to do with a practical algorithm for actually finding the solution of a given system. A very satisfactory algorithm consists merely in constructing successive $I$-minors until an $I$-definite one is reached. The general solution can then be determined by inspection, in accordance with a few simple rules.

The second remark has to do with non-homogeneous inequalities. It is almost obvious that the system

$$
\sum_{j=1}^{\nu} a_{i j} x_{j}+b_{i}>0, \quad(i=1,2, \cdots, \mu)
$$

is essentially equivalent to the system of homogeneous inequalities

$$
\begin{aligned}
\sum_{j=1}^{\nu} a_{i j} x_{j}^{\prime}+b_{i} x^{\prime}{ }_{\nu+1} & >0, \quad(i=1,2, \cdots, \mu), \\
x^{\prime}{ }_{\nu+1} & >0 .
\end{aligned}
$$

Hence the theory of the system (2) can be stated in terms of the matrix

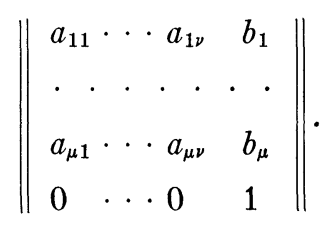

Now shortly after the above theory was formulated, the system (1) was considered from a different point of view by Carver.* He was primarily interested in determining under what conditions the system (1) would admit no solution, that is would be inconsistent. To be sure, an answer to this question is given in Theorem 1 , namely the $I$-rank of the matrix must be zero. But he sought and found a condition of a different and very interesting sort. I shall take the liberty of stating it in somewhat altered form, in order to preserve the analogy

* Annals of Mathematics, vol. 23, p. 212. 
to systems of equations, an analogy which we have had in mind throughout this discussion. You will recall that associated with any given system of linear homogeneous equations there is a second system of similar equations known as the adjoint or associated system, and that the facts relative to the solutions of the two systems are intimately connected. In the problem we are considering it turns out to be convenient to associate with the system of inequalities

$$
\sum_{j=1}^{\nu} a_{i j} x_{j}>0, \quad(i=1,2, \cdots, \mu)
$$

the system of linear equations

$$
\sum_{i=1}^{\mu} a_{i j} y_{i}=0, \quad(j=1,2, \cdots, \nu) .
$$

In order to state nicely the relationship between these two systems, it is desirable to give first some definitions which will be generalized later. A set of real numbers $\left(x_{1}, x_{2}, \cdots, x_{n}\right)$ will be said to be positive if all of its members are positive, or negative if all of its members are negative. In either case it may be said to be definite. Somewhat similar but weaker properties are defined as follows. A set of real numbers is mildly positive $(M$-positive) if at least one of its elements is positive and none are negative; it is mildly negative ( $M$-negative) if at least one element is negative and none are positive. In either case it may be said to be $M$-definite. Carver's theorem can now be stated as follows:

THEOREM 2. A necessary and sufficient condition that the system of inequalities (1) admit no solution $\left(x_{1}, x_{2}, \cdots, x_{\nu}\right)$ is that the associated system of equations ( $\left.1^{\prime}\right)$ admit an $M$-definite solution $\left(y_{1}, y_{2}, \cdots, y_{\mu}\right)$.

The sufficiency of the condition is almost obvious. The necessity requires some argumentation which would be out of place here. Let us rather consider a question which is immediately suggested by Carver's Theorem. That theorem furnishes a criterion for the existence of a mildly definite solution of a system of linear homogeneous equations $\left(1^{\prime}\right)$. Can we by making the condition stronger obtain a criterion for the existence of 
a definite solution of such a system of equations? This turns out to be possible. In order to state the criterion in a form similar to Theorem 2, it is desirable to introduce a modification of the inequality sign. The new symbol $>$ ' is to be interpreted "is somewhere greater than and nowhere less than." Thus the statement $x_{i}>$ '0, $(i=1,2, \cdots, m)$, is equivalent to saying that the numbers $x_{i}$ form an $M$-positive set. We may now state the following theorem.

THEOREM 3. A necessary and sufficient condition that the system of mild inequalities

$$
\sum_{j=1}^{\nu} a_{i j} x_{j}>{ }^{\prime} 0, \quad(i=1,2, \cdots, \mu),
$$

admit no solution $\left(x_{1}, x_{2}, \cdots, x_{\nu}\right)$ is that the associated system of equations

$$
\sum_{i=1}^{\mu} a_{i j} y_{i}=0, \quad(j=1,2, \cdots, \nu),
$$

admit a definite solution $\left(y_{1}, y_{2}, \cdots, y_{\mu}\right){ }^{*}$

The complementary character of the two theorems 2 and 3 is perhaps better exhibited in the following dual statement.

TheOREM 4. A necessary and sufficient condition that the system of linear equations

$$
\sum_{i=1}^{\mu} a_{i j} y_{i}=0, \quad(j=1,2, \cdots, \nu),
$$

admit an $M$-definite (a definite) solution $\left(y_{1}, y_{2}, \cdots, y_{\mu}\right)$ is that the values of the $\mu$ linear forms

$$
\sum_{j=1}^{\nu} a_{i j} x_{j}, \quad(i=1,2, \cdots, \mu)
$$

cannot constitute a definite (an M-definite) set for any values of $\left(x_{1}, x_{2}, \cdots, x_{\nu}\right)$.

It may be of interest in passing to state that a corollary* of Theorem 3 furnishes an easily detectable sufficient condition that a system of linear equations admit a definite solution.

* Annals of Mathematics, vol. 28, pp. 41-42. 
One further remark will conclude what I have to say about algebraic systems. A theory somewhat, though not completely, analogous to that summarized in Theorem 1 for the system of inequalities (1) can be formulated relative to the system of mild inequalities (3). The roles played by the notions $I$-definite and $I$-rank are played by the analogous notions $M$-definite and $M$ rank in the other.

I wish to turn now to some of the extensions and generalizations of the ideas we have been considering. The theory of systems of linear algebraic equations has led in various ways to theories of systems of linear equations in infinitely many unknowns, and to theories of linear integral equations. It is natural to inquire whether the theory of algebraic inequalities can be extended in similar fashion. To put the question, for preliminary examination, in a very general form suggested by the work of Professor E. H. Moore, one may ask what can be said of an inequality of the form

$$
J_{q} \alpha(p, q) \xi(q)>0, \quad(p \text { on range } P),
$$

or

$$
J_{q} \alpha(p, q) \xi(q)>{ }^{\prime} 0, \quad(p \text { on range } P),
$$

where $p$ and $q$ are variables whose ranges are classes of elements $P$ and $Q$ respectively, $\alpha(p, q)$ is a given real-valued function, $\xi(q)$ is the unknown function to be determined, and $J_{q}$ is a linear operator of more or less general nature. The systems of algebraic inequalities which we have considered are obtained from (4) and (5) by specializing the ranges $P$ and $Q$ to be the sets of numbers $1,2, \cdots, \mu$, and $1,2, \cdots, \nu$ respectively, and the operator $J_{q}$ to be the summation operator $\Sigma_{q}$.

Our first remark on this question imposes a great and regrettable limitation, which will later be partially removed. The methods used in developing the algebraic theory involved an algorithm consisting of a finite number of steps corresponding to the finite number of variables $x$. Until other methods are devised, it is impossible to take for the range of the variable $q$ any but a finite set of elements. The operator $J_{q}$ is for the same reason restricted essentially to the finite summation operator $\Sigma_{q}$. The type of inequality which we may hope to treat by methods which are direct generalizations of the algebraic 
theory is therefore much simpler than (4) or (5). Of these two forms, the latter turns out to be the more interesting, as well as the more tractable. Under the limitations just mentioned, it reduces to a form which may be written

$$
\sum_{j=1}^{\nu} \alpha_{j}(p) \cdot \xi_{j}>^{\prime} 0, \quad(p \text { on range } P),
$$

where $\alpha_{j}(p),(j=1,2, \cdots, \nu)$, is a given set of real functions of a general variable $p$, and the set of numbers $\xi_{j}$ is to be determined. In view of the meaning of the symbol $>^{\prime}$, the problem may then be stated as follows: Given a finite set of real functions $\alpha_{j}(p)$ of a variable $p$, the range of which is any class of elements $P$; to determine a linear combination of these functions which is $M$-definite.

A fairly simple generalization of the algebraic theory leads to a satisfactory treatment of this problem. A certain integralvalued function of the set of functions $\left\{\alpha_{j}(p)\right\}$ generalizes the notion of the $M$-rank of the algebraic matrix. Calling it the $M$-rank of the set of functions, we may state the following theorem.*

$A$ necessary and sufficient condition that the set of $\nu$ functions admit an M-definite linear combination is that its $M$-rank be greater than zero. If the $M$-rank is $r(0<r<\nu)$ then there is a subset of $\nu-r+1$ of the functions which admit an M-definite linear combination, but there is no subset of $\nu-r$ functions for which this is true.

A generalized algorithm provides a method for determining an $M$-definite linear combination when one exists.

Of greater interest perhaps are the results obtained in an attempt to generalize the theorem relative to the associated algebraic systems. The adjoint or associated equation of the inequality (6) would be

$$
J_{p} \eta(p) \alpha_{j}(p)=0, \quad(j=1,2, \cdots, \nu),
$$

with $J_{p}$ a suitably restricted linear operator; and the generalized theorem would be to the effect that the inequality (6) admits no solution $\left\{\xi_{i}\right\}$ if and only if the system of equations $\left(6^{\prime}\right)$ admits a positive solution $\eta(p)$. This theorem is un-

* Transactions of this Society, vol. 29, p. 468. 
doubtedly true if the class of functions considered and the linear operator be suitaby restricted; but the problem of determining suitable restrictions so as to retain the greatest possible generality has not been solved. What has been done is to prove the theorem for the two important cases: first, ${ }^{*}$ the case in which the range of $p$ is the infinite sequence $1,2,3, \cdots$, the functions of $p$ considered are infinite sequences of real numbers satisfying certain convergence conditions, and the operator $J_{p}$ is the infinite summation operator; and second, $\uparrow$ the case in which the range of $p$ is a closed interval of real numbers, the functions of $p$ are continuous, and the operator $J_{p}$ is the definite integral over the interval.

The latter of these two theorems is the more interesting, and I shall limit myself to a statement of it. With some changes to alter the emphasis it is as follows.

THEOREM 5. A necessary and sufficient condition that a set of real functions $\alpha_{1}(x), \alpha_{2}(x), \cdots, \alpha_{\nu}(x)$, continuous and linearly independent on an interval $a \leqq x \leqq b$, admit a positive continuous function orthogonal to all of them is that every linear combination of the functions change sign on the interval.

The necessity of the condition is almost obvious; the proof of the sufficiency offers considerable difficulty. The method which was effective was analogous to the method used in the algebraic theory.

I may in passing make a remark similar to one I made in an analogous algebraic situation. While the criterion furnished by Theorem 5 for the existence of a positive function orthogonal to each of a given set of continuous functions may not be practicable, the theorem can be made to yield a sufficient condition $\ddagger$ which is easily detectable when present, and an algorithm for determining such a function in this case.

Now, by the use of Theorem 5 and its analogs, in such fields as it possesses valid analogs, we are able to push somewhat further our study of the general inequality (5). You will recall that so far we have been restricted to cases in which

* Transactions of this Society, vol. 30, p. 440.

$\dagger$ Transactions of this Society, vol. 30, p. 425.

$\ddagger$ Annals of Mathematics, vol. 28, p. 393. 
the range of the variable $q$ is a finite set of elements. We are now able to remove this restriction. Unfortunately we have to replace it by another restriction, which while less stringent, is still regrettable. We shall assume that the kernel $\alpha(p, q)$ has the particular form

$$
\alpha(p, q)=\sum_{i=1}^{m} \phi_{i}(p) \psi_{i}(q),
$$

a restriction which is sometimes described in the integral equation theory by saying that the kernel is of finite rank.

Theorem 6.* A necessary and sufficient condition that the inequality

$$
J_{q} \alpha(p, q) \xi(q)>{ }^{\prime} 0, \quad(p \text { on } P),
$$

admit no solution $\xi$ is that the associated equation

$$
J_{p} \alpha(p, q) \eta(p)=0
$$

$(q$ on $Q)$,

admit a positive solution $\eta$.

The ranges of the variables $p$ and $q$ need not be the same nor even of the same character. The two operators $J_{p}$ and $J_{q}$ may be of different character.

Theorem 6 can be proved on the basis of a few simple postulates concerning the classes of functions considered and the linear operators, postulates which have been proved to hold in the classical instances. In particular, the theorem holds if $p$ and $q$ are ordinary real variables, the functions considered are continuous, and the operators indicate definite integration, in which case (5) and $\left(5^{\prime}\right)$ would have the respective forms

and

$$
\int_{a}^{b} \alpha(x, s) \xi(s) d s>^{\prime} 0, \quad(a \leqq x \leqq b),
$$

$$
\int_{a}^{b} \alpha(s, x) \eta(s) d s=0, \quad(a \leqq x \leqq b) .
$$

There are indications that the serious limitation that the kernel $\alpha$ be of finite rank is not essential to the truth of the

* Transactions of the Royal Society of Canada, vol. 23, p. 141. 
theorem, but no proof has been devised which will apply to a more general type of kernels.

The question naturally arises as to whether a theorem analogous to Theorem 6 holds relative to the strong inequality (4). It is perhaps surprising that the answer is in the negative. A simple example shows that the expected theorem does not hold even for the strong integral inequality analogous to (7).

We come now finally to a type of inequality which would naturally suggest itself first for consideration, but which has been deferred to give place to certain prerequisites. The inequality

$$
\xi(x)+\int_{a}^{b} \alpha(x, s) \xi(s) d s>0, \quad(a \leqq x \leqq b),
$$

may, by analogy with the integral equation theory, be called a linear integral inequality of second kind. The theory of this inequality follows, ${ }^{*}$ with the aid of some of our previous notions, from the theory of the analogous integral equation. For it is indeed equivalent to the equation

$$
\xi(x)+\int_{a}^{b} \alpha(x, s) \xi(s) d s=\pi(x), \quad(a \leqq x \leqq b),
$$

where $\pi(x)$ is an arbitrary positive function.

If the Fredholm determinant of the kernel $\alpha(x, y)$ is different from zero, the general solution of the equation (9) and hence of the inequality (8) may be obtained in the ordinary Fredholm form and will involve the arbitrary positive function $\pi(x)$. If on the other hand, the Fredholm determinant of the kernel $\alpha(x, y)$ is equal to zero, it follows from the Fredholm theory that the equation (9) will admit a solution $\xi(x)$ if and only if the function $\pi(x)$ is orthogonal to every solution $\eta(x)$ of the associated homogeneous equation

$$
\eta(x)+\int_{a}^{b} \alpha(s, x) \eta(s) d s=0,
$$

or what amounts to the same thing if $\pi(x)$ is orthogonal to each of a fundamental set of solutions of (10). Thus a condition

* Transactions of this Society, vol. 30, p. 437. 
is imposed upon the function $\pi(x)$. Suppose a fundamental set of solutions of (10 is before us, say $\eta_{1}(x), \eta_{2}(x), \cdots, \eta_{\nu}(x)$. Can a positive function $\pi(x)$ be determined which is orthogonal to each function of the set? We have the criterion in Theorem 5. Every linear combination of the functions must change sign on the interval in question. But the linear combinations of these functions constitute all the non-trivial solutions of equation (10). Hence we have the following theorem .

THEOREM 7. A necessary and sufficient condition that the integral inequality (8) admit a solution $\xi$ is that every non-trivial solution $\eta$ of the associated integral equation (10) shall change sign.*

It may be noted that this theorem can be stated in a form exactly analogous to Theorem 2 of the algebraic theory.

Time does not warrant a discussion of the generalization of the integral inequality of the second kind. Suffice it to say that it admits of complete generalization $\dagger$ on the basis of Moore's generalized integral equation theory and a few additional postulates.

To summarize briefly the ideas which I have tried to present, two types of systems of algebraic inequalities have been considered: (1) and (3). Each admits a theory somewhat analogous to the classical theory of a system of linear equations. As in the classical theory, so here, there are two points of view: one in which attention is focused on the matrix of coefficients-the rank, the $I$-rank or $M$-rank; the other which finds its interest in the relationship between the given system and an associated system. Theorem 1 is typical for the former and Theorem 2 for the latter.

An attempt has been made to extend the algebraic theory, by way of analogy and generalization, to other types of linear inequalities. The general principle has been to replace the ranges of the variables and the summation operator by more general or at least by different ranges and operators. The attempted generalization has been partially successful. Naturally some

* The case in which the Fredholm determinant is different from zero is compatible with this theorem.

† This Bulletin, vol. 33, p. 695 . 
methods which were effective in the algebraic case are not extensible. Some of the resulting gaps and limitations have been taken care of by alternative methods. Others may be welcomed as outstanding questions, challenges. For example, in Theorem 5 , a statement is made relative to a finite set of functions: can the restriction of finiteness be removed? Again in Theorem 6, the kernel is required to be of finite rank: can this restriction be removed?

For one type of non-algebraic inequality a somewhat complete and satisfactory theory can be given, namely the linear integral inequality of second kind (8) and its generalization in the sense of General Analysis. Some other results, such as Theorem 5, while incomplete from our present point of view, may I hope find points of contact with other investigations in analysis.

The University of Saskatchewan 